\title{
The Mystery behind the Fine Structure Constant Contracted Radius Ratio Divided by the Mass Ratio? A Possible Atomist Interpretation
}

\author{
Espen Gaarder Haug \\ Norwegian University of Life Sciences, Ås, Norway \\ Email: espenhaug@mac.com
}

How to cite this paper: Haug, E.G. (2019) The Mystery behind the Fine Structure Constant Contracted Radius Ratio Divided by the Mass Ratio? A Possible Atomist Interpretation. Journal of High Energy Physics, Gravitation and Cosmology, 5, 899-906. https://doi.org/10.4236/jhepgc.2019.53045

Received: June 6, 2019

Accepted: July 12, 2019

Published: July 15, 2019

Copyright () 2019 by author(s) and Scientific Research Publishing Inc. This work is licensed under the Creative Commons Attribution International License (CC BY 4.0).

http://creativecommons.org/licenses/by/4.0/

\begin{abstract}
This paper examines various alternatives for what the fine structure constant might represent. In particular, we look at an alternative where the fine structure constant represents the radius ratio divided by the mass ratio of the electron, versus the proton as newly suggested by Koshy [1], but derived and interpreted here based on Haug atomism (see [2]). This ratio is remarkably close to the fine structure constant, and it is a dimensionless number. We also examine alternatives including the proton mass divided by the Higgs mass, which appears to be another possible candidate for what the fine structure constant might represent.
\end{abstract}

\section{Keywords}

Fine Structure Constant, Atomism, Electron, Proton, Radius Ratio, Mass Ratio, Higgs Particle

\section{The Fine Structure Constant}

In 1916, Arnold Sommerfeld [3] introduced the fine structure constant in relation to spectral lines. This constant, $\alpha \approx 0.0072973525664$ (2014 CODATA recommended values), plays a vital role in modern physics. Some have suggested that the fine structure constant is related to the ratio of the electron's velocity in the first circular orbit of the Bohr model of the atom to the speed of light in vacuum. An alternative suggestion relates the constant to the Bohr radius by $a_{0}=\frac{\bar{\lambda}_{e}}{\alpha}$, where $\bar{\lambda}_{e}$ is the reduced Compton wavelength of the electron. Furthermore, the 
classical electron radius is given by $r_{e}=\alpha^{2} a_{0}=\alpha \bar{\lambda}_{e}$.

The fine structure constant is also related to the relationship between the charge of an electron and the Planck charge

$$
\alpha=\frac{e^{2}}{q_{p}^{2}}=\frac{\left(\sqrt{\frac{\hbar}{c}} \sqrt{\alpha} \sqrt{10^{7}}\right)^{2}}{\left(\sqrt{\frac{\hbar}{c}} \sqrt{10^{7}}\right)^{2}} .
$$

The Rydberg constant is also a function of the fine structure constant. It has been suggested the fine structure constant is a link between the proton radius and the proton mass, see [4]. We will not comment much on the importance or relevance of these proposed connections. However, we will ask, "Why does the fine structure constant have exactly this 'magical' value?" Or, as stated by Richard Feynman:

It has been a mystery ever since it was discovered more than fifty years ago, and all good theoretical physicists put this number up on their wall and worry about it. Immediately you would like to know where this number for a coupling comes from: is it related to? or perhaps to the base of natural logarithms? Nobody knows. It's one of the greatest damn mysteries of physics. a magic number that comes to us with no understanding by man.

Others have suggested that atomic structures are somehow linked to the golden ratio, which is in turn related to the fine structure ratio (see [5] [6] [7]). The golden angle is given by $\frac{360}{\Phi^{2}} \approx 137.50844$, which is not far from one divided by the fine structure constant: $\frac{1}{\alpha} \approx 137.036$.

In this paper, we will analyze other possible connections to the fine structure constant.

\section{The Contracted Radius Ratio Divided by the Mass Ratio}

In a recent working paper, Koshy [1] suggested that the fine structure constant could be linked to a radius ratio divided by the mass ratio. Here, we build on that idea but in a quite different way. We assume that all matter and energy consist of indivisible particles always moving at the speed of light in the void, as assumed by Haug [2] [8]. Haug's newly introduced atomism theory gives all the same mathematical end results as in Einstein's special relativity theory, when using Einstein-Poincarè synchronized clocks. The theory, moreover, gives upper boundary conditions such as relativistic mass and how close the speed of mass can be to the speed of light.

Each indivisible particle in the electron moves back and forth at the speed of light over the reduced Compton wavelength of the electron. Only at collision is the electron truly a mass. Each collision represents the Planck mass that lasts for one Planck second. This leads to a mass gap of $m_{p} t_{p} \approx 1.17337 \times 10^{-51} \mathrm{~kg}$. The 
electron is the mass gap $\frac{c}{\bar{\lambda}_{e}} \approx 7.763 \times 10^{20}$ times per second, which gives the well-known electron rest-mass (see also [9]). The indivisible particle has a radius equal to the Planck length [10]. This means that the electron has a radius equal to its reduced Compton wavelength when extended ${ }^{1}$. Furthermore, it has only a radius equal to the Planck length when contracted.

The proton-electron mass ratio is $\frac{m_{P}}{m_{e}} \approx 1836.1525$. We could assume the mass of a proton consisted of 1836.1525 electrons (or alternatively 1836). For a moment, we can assume that each of these electrons is a sphere with a radius equal to the Planck length. If we packed these 1836.1525 electrons into a sphere, how much volume would they take up? In 1831, Gauss [11] proved that the most densely one could pack spheres amongst all possible lattice packings was given by

$$
\frac{\pi}{3 \sqrt{2}} \approx 0.74048 \text {. }
$$

In 1611, Johannes Kepler suggested that this was the maximum possible density for both regular and irregular arrangements; this is known as the Kepler conjecture. The Kepler conjecture was supposedly proven in 2014 by Hale [12]. Based on this, the radius of the large sphere consisting of large numbers of densely packed spheres with radius $r$ is approximately given by (see the Appendix)

$$
R \approx \bar{\lambda} \sqrt[3]{\frac{N}{\pi}} \sqrt[6]{18}
$$

This means that the proton's contracted radius is

$$
R \approx r \sqrt[3]{\frac{1836.1525}{\pi}} \sqrt[6]{18} \approx 13.535 r
$$

Next, we will define the contracted radius ratio as

$$
R_{R}=\frac{R}{r}=\frac{r \sqrt[3]{\frac{1836.1525}{\pi}} \sqrt[6]{18}}{r}=\sqrt[3]{\frac{1836.1525}{\pi}} \sqrt[6]{18}
$$

which is the proton's contracted radius divided by the contracted radius of the electron.

If we then divide this contracted radius ratio with the proton's mass divided by the electron's mass, we get a number very close to the fine structure constant:

$$
\alpha \approx \frac{\frac{R}{r}}{\frac{m_{P}}{m_{e}}} \approx 0.0073715
$$

Since $\frac{\bar{\lambda}_{P}}{\bar{\lambda}_{e}}=\frac{m_{e}}{m_{P}}$, we could alternatively have written this in the following form:

${ }^{1}$ And it is extended $\frac{c}{\bar{\lambda}_{e}} \approx 7.763 \times 10^{20}$ times per second. 


$$
\alpha=\frac{R}{r} \frac{\bar{\lambda}_{P}}{\bar{\lambda}_{e}} \approx 13.535 \times \frac{2.10309 \times 10^{-16}}{3.86159 \times 10^{-13}} \approx 0.0073715
$$

Still, this differs somewhat from the fine structure constant $(0.0072973525664$ CODATA 2014); the number is too large. However, the approximation used to calculate the radius of the sphere-packed electrons making up the proton mass will actually slightly overestimate the radius of the sphere-packed sphere. This is because the sphere-packed sphere's outer surface is not smooth but rather jagged. We could measure the average radius of the sphere-packed spheres by measuring the radius from the inside radius and the outside radius and divide by two (see Figure 1).

Figure 1 illustrates how we account for the sphere-packed sphere's jagged surface, namely the diameter of the average of the blue line and the green line. To find the green line, we can use the Pythagorean theorem to discern the distance, as shown in the lower part of Figure 1. If one properly adjusts for the jagged surface of the hypothetical sphere-packed sphere, it then seems that the radius of the large sphere should be very close to 13.4012 relative to the electron's radius (the contracted radius ratio).

$$
\begin{aligned}
R_{R} & \approx \frac{\sqrt[3]{\frac{N}{\pi}} \sqrt[6]{18}+\left(\sqrt[3]{\frac{N}{\pi}} \sqrt[6]{18}-1+(\sqrt{3}-1)\right)}{2}=\frac{2 \sqrt[3]{\frac{N}{\pi}} \sqrt[6]{18}+\sqrt{3}-2}{2} \\
& =\sqrt[3]{\frac{N}{\pi}} \sqrt[6]{18}+\sqrt{\frac{3}{4}}-1=13.4012
\end{aligned}
$$

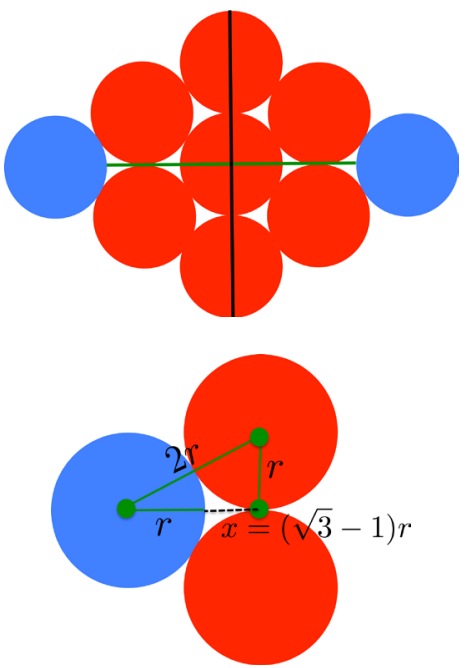

Figure 1. The figure illustrates the contracted radius of a sphere (here we only see a cross-section of the sphere). As a sphere-packed sphere's surface must be jagged, a good approximation for the radius is found by taking half the average of the black-lined diameter and the green-lined diameter. To find the green-lined diameter, we need to use the Pythagorean theorem, as illustrated in the subfigure below. The contracted proton radius can, in the same way, be seen as 1836 sphere-packed spheres. The green-lined diameter is equal to the black-lined diameter minus $2 r-2(\sqrt{3}-1) r \approx 0.54 r$, where $r$ is the radius of the small spheres, which, based on recent developments in mathematical atomism, must be $r=l_{p}$, which is the Planck length. 
And from this, we can calculate the fine structure constant by dividing the contracted radius ratio by the mass ratio:

$$
\alpha=\frac{R_{R}}{\frac{m_{P}}{m_{e}}} \approx \frac{13.4012}{1836.152} \approx 0.00729854 .
$$

This also means that the fine structure constant can be represented by the contracted radius ratio multiplied by the ratio of the reduced Compton wavelengths. The calculated value is extremely close compared to $\alpha_{c}=0.0072973525664$, which is the fine structure constant given by CODATA 2014. The difference between the two numbers is close to $\frac{\alpha_{c}-\alpha}{\alpha_{c}}=0.0161 \%$. We do not claim that this is what the fine structure constant must represent, but again it is interesting that this is a dimensionless number.

Alternatively, we could have used the classical electron radius $r_{e}=\frac{1}{4 \pi \varepsilon_{0}} \frac{e^{2}}{m_{e} c^{2}}=\alpha \bar{\lambda}_{e} \approx 2.81794 \times 10^{-15}$. The classical electron radius ${ }^{2}$ divided by the reduced Compton wavelength of the proton $^{3}$ is:

$$
\text { Radius ratio }=\frac{\alpha \bar{\lambda}_{e}}{\bar{\lambda}_{P}} \approx 13.39905249,
$$

and the fine structure constant is given by

$$
\alpha \approx \frac{\frac{\alpha \bar{\lambda}_{e}}{\bar{\lambda}_{P}}}{\frac{m_{P}}{m_{e}}}=\frac{\frac{\alpha \bar{\lambda}_{e}}{\bar{\lambda}_{P}}}{\frac{\hbar}{\bar{\lambda}_{P}} \frac{1}{c}}=\frac{13.39905249}{1836.152}=\alpha \times 1 \approx 0.007297353 .
$$

In this case, it is the electron's radius divided by the proton's radius, while in the above analysis it was the proton's contracted radius divided by the electron's contracted radius (according to the atomism model.). We believe that the classical electron radius likely does not exist in a physical sense; it is just an imaginary unit that has the fine structure constant embedded. On the other hand, the contracted radius ratio is something that possibly exists if the depth of reality is atomism.

When it comes to the relationship between the classical electron radius and the radius of the proton or neutron and their mass ratio, Koshy has, in a recent piece [1], suggested a similar relationship as a possible interpretation of the fine structure constant. However, we believe that the Haug atomist model has greater explanatory power, in terms of what is gained from Einstein's special relativity

\footnotetext{
${ }^{2}$ The previous analysis also means we can write the classical electron radius as

$r_{e}=\frac{R}{r} \bar{\lambda}_{P} \approx 13.4012 \times \bar{\lambda}_{P}$.

${ }^{3}$ Here using CODATA (divided by $2 \pi$ to get the reduced form): $\bar{\lambda}_{P} \approx 2.103089101 \times 10^{-16}$ and $\bar{\lambda}_{e} \approx 3.861592677 \times 10^{-13}$.
} 
mathematical result that is obtained when using Einstein-Poincaré synchronized clocks. This theory also seems to make more sense when the diameter of the indivisible particle is the Planck length and its mass is the Planck mass. Moreover, a series of infinity problems are elegantly removed via the Haug model.

On its own, this result could be seen as nothing more than numerology. Yet the result of the Haug model is particularly interesting when seen in light of recent developments in mathematical atomism. Haug's mathematical atomism model is very simple and thus far has been shown to yield the same mathematical result as Einstein's special relativity theory when using Einstein-Poincaré synchronized clocks.

Interestingly, If the fine structure constant truly could be represented by the radius ratio divided by the mass ratio, then it would not be affected by relativistic effects.

\section{Proton Mass Divided by Higgs Mass}

The 2014 CODATA-recommended proton mass is $1.672621898 \times 10^{-27} \mathrm{~kg}$. That is equal to about $938.2721137 \mathrm{MeV} / c^{2}$. On 4 July 2012, CMS announced the discovery of a previously unknown boson with mass $125.3 \pm 0.6 \mathrm{MeV} / c^{2}$, see [13]. There is still considerable uncertainty about the mass of the Higgs boson [14]. For a moment, assume the Higgs mass is approximately $128,577.056 \mathrm{MeV} / \mathrm{c}^{2}$. In this case, the proton mass divided by the Higgs mass would be close to identical to the fine structure constant, and it would be a dimensionless constant:

$$
\alpha \approx \frac{m_{P}}{m_{H}} \approx \frac{938.2721137}{128577.056} \approx 0.007297353,
$$

while a Higgs mass of $125.3 \mathrm{GeV}$ would give a fine structure constant of

$$
\alpha \approx \frac{m_{P}}{m_{H}} \approx \frac{938.2721137}{125300} \approx 0.007488205 .
$$

Still, this suggested value of the Higgs boson seems too far away from what it should in order to be related to the fine structure constant. Moreover, it is also in relation to electrons where the fine structure constant seems to be most important.

\section{Summary}

The fine structure constant plays an important role in modern physics. Yet there continues to be a mystery concerning exactly what it represents and why it has the mystical value it does. In this paper, we have suggested two new possibilities for what the fine structure constant may represent. It could be related to what we would call the contracted radius ratio of the electron versus the proton divided by the mass ratio, an idea closely related to the work of Koshy [1]. The contracted radius ratio is given from sphere packing of Planck diameter spheres and adjusting for this sphere-packed sphere's jagged surface. This new ratio 
seems to be extremely close to the fine structure constant given by CODATA. Alternatively, we have suggested that the fine structure constant could be related to the Higgs mass over the proton mass, but this suggestion seems to give a fine structure constant considerably distant from the one given by CODATA.

We have in this paper not concluded what the fine structure constant truly represents. But we believe that the speculative idea that spins off from atomism deserves further investigation.

\section{Acknowledgements}

Thanks to Richard Whitehead and Victoria Terces for assisting with manuscript editing. Thanks to Thijs van den Berg for a useful discussion on the wilmott.com forum in relation to circle geometry.

\section{Conflicts of Interest}

The author declares no conflicts of interest regarding the publication of this paper.

\section{References}

[1] Koshy, P. (2017) Fine Structure Constant a Mystery Resolved.

[2] Haug, E.G. (2014) Unified Revolution, New Fundamental Physics. E.G.H. Publishing, Oslo.

[3] Sommerfeld, A. (1916) On the Quantum Theory of Spectral Lines. Annals of Physics, 51.

[4] Haug, E.G. (2018) An Interesting Mathematical Relation between the Proton Mass, the Proton Radius, the Fine Structure Constant, the Compton Wavelength and the Hagedorn Temperature. Journal of High Energy Physics, Gravitation and Cosmology, 5, 438-441. https://doi.org/10.4236/jhepgc.2019.52025

[5] Heyrovska, R. and Narayan, S. (2005) Fine-Structure Constant, Anomalous Magnetic Moment, Relativity Factor and the Golden Ratio That Divides the Bohr Radius. https://arxiv.org/pdf/physics/0509207.pdf

[6] Sherbon, M.A. (2012) Wolfgang Pauli and the Fine-Structure Constant. Journal of Science, 2, 148-154. https://doi.org/10.2139/ssrn.1934553

[7] Heyrovska, R. (2013) Golden Ratio Based Fine Structure Constant and Rydberg Constant for Hydrogen Spectra. International Journal of Sciences, 2, 28-31.

[8] Haug, E.G. (2016) The Panck Mass Particle Finally Discovered! The True God Particle! Good Bye to the Point Particle Hypothesis! http://vixra.org/abs/1607.0496

[9] Haug, E.G. (2017) The Mass Gap, Kg, the Planck Constant and the Gravity Gap. http://vixra.org

[10] Planck, M. (1906) Vorlesungen uber die Theorie der Warmestrahlung. J.A. Barth, Leipzig, 163.

[11] Gauss, C.F. (1831) Besprechung des buchs von l.a. seeber: Intersuchungen ber die eigenschaften der positiven ternaren quadratischen formen usw. Gttingsche Gelehrte Anzeigen.

[12] Hales, T., et al. (2014) Flyspeck-Announcing Completion. https://code.google.com/archive/p/flyspeck/wikis/AnnouncingCompletion.wiki

[13] CMS Collaboration (2012) Observation of a New Boson with a Mass near $125 \mathrm{GeV}$. 
Cms-Pas-Hig-12-020.

[14] Aad, G., et al. (2015) Combined Measurement of the Higgs Boson Mass in $p p$ Collisions at $\sqrt{\mathrm{s}}=7$ and $8 \mathrm{TeV}$ with the ATLAS and CMS Experiments. Physical Review Letters, 114, Article ID: 191803.

\section{Appendix: Radius of Spheres Constructed from a Large Number of Small Spheres}

Assuming small spheres with radius $r$, the volume of such a sphere is

$$
V=\frac{4}{3} \pi r^{3} .
$$

When we pack the Planck spheres as densely as possible, they will occupy a volume of

$$
V_{t}=\frac{\frac{4}{3} \pi l_{p}^{3}}{\frac{\pi}{3 \sqrt{2}}}=l_{p}^{3} \sqrt{32}
$$

The total volume is then $N V_{t}$. This means we need a larger sphere with radius

$$
\begin{gathered}
N V_{t}=\frac{4}{3} \pi R^{3} \\
R=\sqrt[3]{\frac{\frac{3}{4} N V_{t}}{\pi}} \\
R=\sqrt[3]{\frac{\frac{3}{4} N r^{3} \sqrt{32}}{\pi}} \\
R=r \sqrt[3]{\frac{N}{\pi} \sqrt[6]{18}}
\end{gathered}
$$

It is important to be aware that this formula will only be a good approximation for a very large number of spheres. In the case of a proton, we will assume it consists of 1836 spheres, which is a number of spheres where this formula should be quite accurate. 